\title{
Theory of Spin Polarization Phenomena in Atomic and Molecular Photoeffects
}

\author{
M. Ya. Agre \\ International Solomon University, Kiev, 01135 Ukraine \\ National University Kyiv Mohyla Academy, Kiev, 04070 Ukraine \\ e-mail:markag@aport2000.ru \\ Received September 5, 2005
}

\begin{abstract}
A compact invariant expression for the angular distribution of photoelectrons formed as a result of photoionization of axisymmetrically polarized atoms and molecules, including optically active molecules, is derived from the general symmetry considerations and using a recently developed bipolar harmonic reduction technique. In the angular distribution of photoelectrons, as well as in the total photoionization cross section, the dependence on all the geometric parameters is separated completely. The polarization of ionizing radiation can be arbitrary, with the partial polarization being specified by the Stokes parameters. The expression for the angular distribution of photoelectrons escaping from an atomic-molecular system oriented in the first order also determines the structure of the expression for the photoionization cross section of an unpolarized system with allowance for the spin polarization of a photoelectron or a photoion with the spin $1 / 2$. The roles played by circular dichroism, chirality, etc., in the process of photoionization of oriented and aligned systems are analyzed in detail.
\end{abstract}

PACS numbers: $32.80 . \mathrm{F}$

DOI: $10.1134 / \mathrm{S} 0030400 \mathrm{X} 06090050$

\section{INTRODUCTION}

The photoeffect is one of the most studied elementary photoprocesses, and, in this sense, it can be considered as classical. The role played by this phenomenon in the development of quantum notions about the nature of light is well known.

The theoretical investigation of the atomic (molecular) photoeffect, as well as of other elementary processes, can be significantly simplified upon separation of geometric and dynamic parameters in expressions for the corresponding cross sections. This problem is solved by the methods of angular momentum algebra. The dynamic parameters are determined by the internal structure of a system, and their calculation is a rather complex problem, which is still not solved completely. Therefore, it is important to have at hand simple analyzable expressions for the cross sections that allow one to find the dynamic parameters from experimental measurements.

As is well known, the photoionization cross section of a freely orientable (unpolarized) atom, which determines the angular distribution of photoelectrons under conditions where the spin orientation of a photoelectron is not measured, contains in the dipole approximation two dynamic parameters: the photoeffect total cross section $\sigma$ and the angular-asymmetry scalar parameter $\beta$ [1] (see formula (7) below). In standard approximations used in atomic calculations [2], the dynamic parameters $\sigma$ and $\beta$ are expressed in terms of five independent quantities: the three dipole matrix elements of bound-free transitions and two phase differences of photoelectron scattering (see, e.g., [2, 3]). Consequently, it is fundamentally impossible to determine all the five independent atomic parameters from the experimental investigation of the angular distribution of photoelectrons formed upon photoionization of unpolarized atoms, i.e., to realize the so-called complete experiment.

For the complete experiment to be realized, more subtle measurements should be performed. Thus, in [35], it was suggested to measure additionally the spin polarization of photoelectrons, which makes it possible to determine three additional dynamic parameters from experimental data. It has proved possible, at least for linear and circular polarizations of ionizing radiation, to write the corresponding expression for the photoeffect cross section in a compact invariant form convenient for analysis [3]. However, it has recently been found that there exists a relation between these three additional dynamic parameters and the angular asymmetry parameter $\beta$ [6]; hence, in order to perform the complete experiment, it is also necessary to measure the spin polarization of a photoion [7].

As was shown by Klar and Kleinpoppen [8], an alternative possibility for performing the complete experiment consists of investigating the angular distribution of photoelectrons escaping from polarized atoms. An atom is polarized upon light absorption, col- 
lisions, and other processes disturbing the equilibrium population of its magnetic sublevels. A special method of polarization of atoms, namely, optical pumping, has been developed (see reviews $[9,10])$. The state of an axisymmetrically polarized system is specified by $2 j$ independent quantities $(j$ is the quantum number of the total angular momentum), for which so-called state multipoles can be taken [11]. The photoeffect cross section is linear in state multipoles and contains a considerably greater number of dynamic parameters, which can be found from experimental measurements. In turn, these parameters are expressed in terms of the five independent parameters indicated above (the dipole matrix elements and the scattering phase differences).

In the expression obtained in [8] for the photoionization cross section of a polarized atom, the geometric and dynamic parameters were separated. However, a dependence on the direction of motion of a photoelectron and on the direction of the polarization axis of an atomic target appeared in the expression for the angular distribution of photoelectrons via bipolar harmonics [12] (the photoionization cross section taking into account the photoelectron spin polarization contained tripolar harmonics). In addition, the expression for the cross section was written in a noninvariant form in a coordinate system whose $z$ axis coincided either with the direction of propagation of circularly polarized or unpolarized light or with the direction of the linear polarization of the light wave. For such a representation of the cross section, the separation of the explicit dependence in it on all the vectors characterizing the process proved to be not a simple task, which reduced to expressing the corresponding bipolar harmonics in a particular coordinate system. In [8], this procedure was realized for the simplest cases, in which several of the first state multipoles are different from zero. As a result, cumbersome and difficult-to-analyze formulas were obtained, which seemingly made an oppressive impression on experimenters. In all subsequent theoretical studies on photoionization of polarized atoms known to the author that were performed up to 1996, these difficulties were not overcome (see, e.g., [13-16]).

The situation changed radically after the publishing of a seminal paper by Manakov et al. [17], in which a method of reduction of bipolar harmonics (and, in the general case, multipolar harmonics) to the simplest bipolar harmonics with minimal possible ranks of internal spherical functions was developed. The method developed in that study can be applied to a wide range of problems in atomic and nuclear physics. To illustrate the method proposed, the authors of [17] considered several examples, including the problem of photoionization of polarized atoms, which is the subject of this study. They showed that, in fact, a compact invariant expression for the cross section of the process, in which the dependence on all the vectors involved is separated in the form of scalar and triple scalar products, can be obtained from the symmetry considerations alone, without invoking any approximation used in atomic calculations.

The objective of this study is to develop further the application of the method of [17] in the theory of photoionization of polarized atomic systems. First of all, the results of [17] are generalized to the case of a partial polarization of ionizing radiation. Then, the theory of photoionization of optically active molecules is developed. The structure of the photoionization cross section of optically inactive molecules does not differ from that of atoms. At the same time, the expression for the photoionization cross section of optically active (chiral) molecules contains additional terms and, correspondingly, a number of new effects are observed in the course of ionization.

The paper is organized as follows. In Section 2, the structure of the expression for the dipole photoionization cross section of a polarized atomic-molecular system is determined from the general symmetry considerations. Then, compact invariant expressions are obtained that determine the angular distribution of photoelectrons formed upon photoionization by polarized radiation of polarized atoms and achiral molecules (Section 3) and of optically active molecules (Section 4). The expressions for bipolar harmonics reduced according to the method of [17] that are used in this study are given in the Appendix. In Section 5, we discuss the structure of the expression for the total photoionization cross section, which can be obtained both from the general symmetry considerations and by direct integration of the angular distribution of photoelectrons. Then, in Section 6, the dependence on the Stokes parameters of ionizing radiation is separated in the formulas obtained and the expressions for the cross sections become applicable to the general case of a partial polarization of a photon. To illustrate the simplicity and efficiency of the method developed in this study, we consider the photoionization of atomic-molecular systems oriented in the first order (Section 7) and aligned in the first order (Section 8) and analyze the effects of circular dichroism, the contribution of chirality, and so on. Note that the expressions for the photoionization cross section of an oriented system presented in Section 7 also describe the structure of the expression for the photoionization cross section of an unpolarized system taking into account the spin polarization of a photoelectron or a photoion if the spin of the latter is equal to $1 / 2$.

Therefore, it can be stated that this study is devoted to the theory of spin polarization phenomena related to the photoeffect that involve the spin of an atom (molecule), the spin of a photoelectron, and the spin of a photon. 


\section{THE GENERAL STRUCTURE OF THE EXPRESSION}

FOR THE PHOTOIONIZATION CROSS SECTION OF A POLARIZED ATOMIC-MOLECULAR

\section{SYSTEM}

In the general case, a polarized atom (molecule) resides in a quantum-mechanical state that is mixed with respect to the projections of the angular momentum and is described by the density matrix. The decomposition of the density matrix into irreducible components, which are termed state multipoles (statistical tensors) [11], makes it possible to distinguish different types of polarization. The state multipole $\rho_{K Q}$, where $K=0,1,2, \ldots, 2 j$ and $Q=-K,-K+1, \ldots, K$ is a $K$ th-rank irreducible tensor. In the absence of polarization, the system is spherically symmetric; therefore, only the zeroth-rank state multipole (i.e., the scalar)

$$
\rho_{00}=(2 j+1)^{-1 / 2}
$$

differs from zero. If at least one odd-rank state multipole is nonzero, the system is referred to as an oriented system, and, if at least one even-rank state multipole is nonzero, the system is termed an aligned system. To specify the particular type of polarization, I, as in my previous studies on light scattering $[18,19]$, will distinguish orientation and alignment of different orders. Thus, if the vector $\rho_{1 Q}$, which is proportional to the average angular momentum of the polarized system [11],

$$
\rho_{1 Q}=(-1)^{Q} \sqrt{3}[j(j+1)(2 j+1)]^{-1 / 2} \bar{j}_{-Q},
$$

differs from zero, we will speak of first-order orientation; if the tensor $\rho_{3 Q}$ is nonzero, the orientation will be second-order; and so on. Correspondingly, alignment of the first, second, etc., order is associated with a nonzero state multipole of the second, fourth, etc., rank.

As in all previous studies on photoionization of polarized atoms, ${ }^{1}$ we will restrict ourselves to the important particular case of an axisymmetric polarization, whose symmetry axis is specified by the vector $\mathbf{n}$. Such a polarization obviously arises if an external polarizing perturbation is axisymmetric (for example, unpolarized electromagnetic radiation or radiation that is polarized circularly or linearly). In the case of an axisymmetric polarization, only the zeroth components $\rho_{K 0}=\rho_{K}^{n}$ of all the state multipoles differ from zero in a coordinate system whose $z$ axis is directed along $\mathbf{n}$; as a result, the density matrix of the polarized atom proves to be diagonal with respect to the projections of the angular momentum $M$ onto the direction $\mathbf{n}$ and the state of the atom is an incoherent mixture of states with different $M$ [11]. It should be noted here immediately that

\footnotetext{
${ }^{1}$ For brevity, below, we will speak of an atom implying both an atom and a molecule. The results pertaining only to optically active molecules will be described separately; therefore, such a terminology should not cause ambiguity.
}

all $\rho_{K}^{n}$ of odd rank $K$ change their sign upon time reversal and upon spatial inversion (in the latter case, the vector $\mathbf{n}$ is changed to $-\mathbf{n}$ ); i.e., they are $T$-odd pseudoscalars, whereas all even-rank $\rho_{K}^{n}$ are $T$-even scalars. Using the law of transformation of state multipoles upon rotations, we can find the state multipoles and the elements of the density matrix in an arbitrary coordinate system [11],

$$
\begin{gathered}
\rho_{K Q}=\sqrt{\frac{4 \pi}{2 K+1}}(-1)^{Q} Y_{K,-Q}(\mathbf{n}) \rho_{K}^{n}, \\
\left\langle j m|\hat{\rho}| j m^{\prime}\right\rangle \\
=\sum_{K, Q}(-1)^{j-m}(2 K+1)^{1 / 2}\left(\begin{array}{ccc}
j & j & K \\
m & -m^{\prime} & -Q
\end{array}\right) \rho_{K Q} \\
=\sqrt{4 \pi} \sum_{K, Q}(-1)^{j-m-Q}\left(\begin{array}{ccc}
j & j & K \\
m-m^{\prime} & Q
\end{array}\right) Y_{K Q}(\mathbf{n}) \rho_{K}^{n},
\end{gathered}
$$

where $Y_{K Q}(\mathbf{n})$ is a spherical function.

In order to determine the general structure of the expression for the photoeffect cross section, we will proceed from the fact that, in the first order of the perturbation theory, the probability of transition of a polarized atom to the state $|f\rangle$ under the action of the perturbation $\hat{V}$ is proportional to the following expression:

$$
\sum_{m, m^{\prime}, \ldots}\left\langle f|\hat{V}| v_{i} j m\right\rangle\left\langle j m|\hat{\rho}| j m^{\prime}\right\rangle\left\langle v_{i} j m^{\prime}\left|\hat{V}^{+}\right| f\right\rangle .
$$

Here, $v_{i}$ denotes the set of the internal quantum numbers of the atom in the initial state, which already does not contain $j$ and $m$, and the dots under the summation sign refer to the possible summation over the quantum numbers of the final state $|f\rangle$. Since we are interested only in the angular distribution of photoelectrons, the summation is performed over the projections of the photoelectron and photoion spins. In this case, in the differential photoionization cross section, which determines the angular distribution, the dependence on the direction of motion of a photoelectron (we will set this direction by the unit vector $\mathbf{p}$ ) is retained. The dependence on $\mathbf{p}$ vanishes upon going to the total cross section of the process, in which the integration is performed over all the directions $\mathbf{p}$.

For the time being, the electromagnetic radiation ionizing the atom is assumed to be completely polarized, with its polarization being specified by the unit vector e. In the dipole approximation, the operator $\hat{V}$ in (4) determining the interaction of the atom with the electromagnetic radiation is proportional to (ed),

$$
\hat{V} \sim(\mathbf{e d})=\sum_{q}(-1)^{q} e_{q} d_{-q},
$$


where $\mathbf{d}$ is the dipole moment operator of the atom and $a_{q}, q=0, \pm 1$, are the spherical components of the vector $\mathbf{a}[12]$.

From formulas (3) and (4), it follows that the photoionization cross section is linear in state multipoles $\rho_{K}^{n}$ and can be written as

$$
\frac{d \sigma(\mathbf{n})}{d \Omega}=\frac{d \sigma}{d \Omega}+\frac{1}{4 \pi} \sum_{K=1}^{2 j} \rho_{K}^{n} \frac{d \sigma_{K}(\mathbf{n})}{d \Omega} .
$$

The first term in (6) determines the angular distribution of photoelectrons formed upon photoionization of unpolarized atoms; therefore, it can be immediately written in the standard form ${ }^{2}$ (see also the next section)

$$
\frac{d \sigma}{d \Omega}=\frac{\sigma}{4 \pi}\left(1+\beta \frac{3|\mathbf{e p}|^{2}-1}{2}\right) .
$$

It remains to clarify how the remaining terms of (6), which determine the polarization additions to the angular distribution of photoelectrons, depend on the geometric parameters, i.e., on the vectors $\mathbf{e}, \mathbf{n}$, and $\mathbf{p}$.

We will show that the dependence of all the terms in (6) on the geometry can be determined from the symmetry considerations alone. Indeed, from the spherical coordinates of the polarization vector $\mathbf{e}$, one can compose according to the general rule [12] the irreducible tensors of the ranks $r=0,1$, and 2 ,

$$
\left\{\mathbf{e} \otimes \mathbf{e}^{*}\right\}_{r p}=\sum_{q, q^{\prime}} C_{1 q 1 q^{\prime}}^{r p} e_{q}\left(e^{*}\right)_{q^{\prime}},
$$

where $C_{b \beta c \gamma}^{a \alpha}$ is the Clebsch-Gordan coefficient. Let us introduce the scalar product of two irreducible tensors,

$$
\begin{aligned}
& \left(B_{r} D_{r}\right)=\sum_{q}(-1)^{q} B_{r, q} D_{r,-q} \\
& =(-1)^{r} \sqrt{2 r+1}\left\{B_{r} \otimes D_{r}\right\}_{0}
\end{aligned}
$$

and the irreducible tensors $T_{r}$ of the $r$ th rank, proportional to the tensors defined by (8) [17],

$$
T_{r}=(-1)^{1-r} \sqrt{\frac{3(r+1)}{2 r+1}}\left\{\mathbf{e} \otimes \mathbf{e}^{*}\right\}_{r} .
$$

The zeroth- and first-rank irreducible tensors (8) are expressed in terms of the scalar and vector products of the corresponding vectors [12],

$$
\begin{aligned}
T_{0}=\left(\mathbf{e e}^{*}\right) & =1, \quad T_{1}=i\left[\mathbf{e}, \mathbf{e}^{*}\right]=\xi \mathbf{k}, \\
T_{2} & =-\frac{3}{\sqrt{5}}\left\{\mathbf{e} \otimes \mathbf{e}^{*}\right\}_{2},
\end{aligned}
$$

\footnotetext{
${ }^{2}$ In the case of unpolarized optically active molecules, the expression for the angular distribution of photoelectrons should contain one more term that determines the effect of circular dichroism $[3$, 20] (see formula (21) below).
}

where $\mathbf{k}$ is the unit vector defining the direction of propagation of the electromagnetic wave and the parameter

$$
\xi=i\left(\mathbf{k}\left[\mathbf{e}, \mathbf{e}^{*}\right]\right)
$$

specifies the degree of its circular polarization (for the right-hand or left-hand circular polarization, $\xi= \pm 1$, while, for the linear polarization, $\xi=0$ ). Reversing relation (8), one can express $e_{q}\left(e^{*}\right)_{q^{\prime}}$ in terms of the components of the irreducible tensors $T_{r}$. Taking into account expression (4) for the transition probability, we obtain that the expression for the differential photoionization cross section should have the following structure:

$$
\frac{d \sigma}{d \Omega}=\sum_{r=0}^{2}\left(A_{r}(\mathbf{n}, \mathbf{p}) T_{r}\right),
$$

where, in view of the fact that the cross section is scalar, the quantities $A_{r}(\mathbf{n}, \mathbf{p})$ should be irreducible tensors of the $r$ th rank composed of the vectors $\mathbf{n}$ and $\mathbf{p}$. Expression (3) for the density matrix of the polarized atom shows that $A_{r}$ is linear in $\rho_{K}^{n} Y_{K Q}(\mathbf{n})$; consequently, the general structure of these irreducible tensors is obvious:

$$
A_{r}(\mathbf{n}, \mathbf{p})=\sum_{K=0}^{2 j} \rho_{K}^{n} \sum_{l=|K-r|}^{K+r} A_{K l}^{r}\left\{Y_{K}(\mathbf{n}) \otimes Y_{l}(\mathbf{p})\right\}_{r} .
$$

Here,

$$
\left\{Y_{K}(\mathbf{n}) \otimes Y_{l}(\mathbf{p})\right\}_{r m}=\sum_{Q, M} C_{K Q l M}^{r m} Y_{K Q}(\mathbf{n}) Y_{l M}(\mathbf{p})
$$

is the bipolar harmonic (the irreducible tensor composed of two spherical functions) [12] and $A_{K l}^{r}$ are the atomic dynamic parameters.

Formulas (11), (12), and (10) determine the dependence of the photoionization cross section (correspondingly, all terms in (6)) on the geometric parameters. To represent this dependence in a compact invariant form, we will apply the method of reduction of bipolar harmonics developed in [17]. We will consider separately optically inactive atomic-molecular systems and optically active molecules.

\section{ANGULAR DISTRIBUTION OF PHOTOELECTRONS FORMED UPON PHOTOIONIZATION OF POLARIZED ATOMS AND POLARIZED OPTICALLY INACTIVE MOLECULES}

The dynamic parameters $A_{K l}^{r}$ of an atom ${ }^{3}$ or an optically inactive molecule are true scalars; i.e., they remain invariant under the operation of spatial inversion. At the same time, the product $\rho_{K}^{n} Y_{K Q}(\mathbf{n})$ in

\footnotetext{
${ }^{3}$ In this section, unless otherwise stated, the word atom refers both to an atom and to a molecule having no optical activity.
} 
decomposition (12) is also invariant under spatial inversion (is $P$-even). Therefore, the spherical function $Y_{I M}(\mathbf{p})$ in bipolar harmonic (13) of this decomposition should also be $P$-even; in other words, the rank $l$ of the spherical function should be even. This additional symmetry condition, together with the formulas for bipolar harmonics given in the Appendix, makes it possible to obtain compact expressions for all the quantities appearing in the angular distribution of photoelectrons (6).

Initially, an expression for the angular distribution of photoelectrons formed upon photoionization of unpolarized atoms will be presented, which contains only two dynamic parameters: $A_{00}^{0}$ and $A_{02}^{2}$. Using formulas (A.3) and (A.7), in which one should set $K=0$ (in this simple case, one can do without these formulas, applying the definition of bipolar harmonics), and expressions (10) for the irreducible tensors $T_{r}$, one can obtain from (11) and (12) the following expression:

$$
\frac{d \sigma}{d \Omega}=\frac{1}{4 \pi(2 j+1)^{1 / 2}}\left[A_{00}^{0}+\left(1-3|\mathbf{p e}|^{2}\right) \sqrt{\frac{3}{2}} A_{02}^{2}\right] .
$$

In deriving (14), we also used the identity [12]

$$
\begin{gathered}
\left\{\{\mathbf{a} \otimes \mathbf{b}\}_{2} \otimes\{\mathbf{c} \otimes \mathbf{d}\}_{2}\right\}_{0} \\
=(1 / \sqrt{5})[(1 / 2)(\mathbf{a c})(\mathbf{b d})+(1 / 2)(\mathbf{a d})(\mathbf{b c}) \\
-(1 / 3)(\mathbf{a b})(\mathbf{c d})],
\end{gathered}
$$

which expresses one of the scalar products in (11) (see (9) at $r=2$ ) in terms of the scalar products of the vectors contained in it, and took into account the explicit expression (1) for $\rho_{0}^{n}$. By comparing (14) with the standard form (7) of the angular distribution of photoelectrons, we can relate $\sigma$ and $\beta$ with the dynamic atomic parameters introduced,

$$
\sigma=A_{00}^{0}(2 j+1)^{-1 / 2}, \quad \beta=-\sqrt{6} A_{02}^{2} / A_{00}^{0} .
$$

Below, in a similar fashion, we will write the expressions for the quantities $d \sigma_{K} / d \Omega$ contained in (6) separately for odd $K$, for which these quantities determine the contribution of the orientation of an atom to the photoionization process, and for even $K$, for which they determine the contribution of the alignment.

\section{Odd K(Orientation)}

With the help of formulas (A.5) and (A.6), reducing bipolar harmonics; definition (10) of tensors $T_{r}$; and identities (9) and (15), we can obtain from (11) and (12) the following expression for the sought quantities:

$$
\begin{gathered}
d \sigma_{K}(\mathbf{n}) / d \Omega=a_{K}^{(1)} \xi(\mathbf{k n})+a_{K}^{(2)} \xi(\mathbf{k p}) \\
+a_{K}^{(3)} \operatorname{Re}\left[(\mathbf{n e})\left([\mathbf{p}, \mathbf{n}] \mathbf{e}^{*}\right)\right]+a_{K}^{(4)} \operatorname{Re}\left[(\mathbf{p e})\left([\mathbf{n}, \mathbf{p}] \mathbf{e}^{*}\right)\right]
\end{gathered}
$$

Here, the scalar functions $a_{K}^{(i)}(\mathbf{n p})$ are introduced, which are defined as follows:

$$
\begin{gathered}
a_{K}^{(1)}=\sqrt{3}\left(\frac{A_{K, K+1}^{1}}{\sqrt{K+1}}+\frac{A_{K, K-1}^{1}}{\sqrt{K}}\right) P_{K}^{(1)}(\mathbf{n p}), \\
a_{K}^{(2)}=-\sqrt{3}\left[\frac{A_{K, K+1}^{1}}{\sqrt{K+1}} P_{K+1}^{(1)}(\mathbf{n p})+\frac{A_{K, K-1}^{1}}{\sqrt{K}} P_{K-1}^{(1)}(\mathbf{n p})\right] \\
\times\left(\frac{a_{K}^{(3)}=3\left[\frac{2}{K(K+1)}\right]^{1 / 2}}{\sqrt{K+2}}+\frac{\operatorname{Im} A_{K, K-1}^{2}}{\sqrt{K-1}}\right) P_{K}^{(2)}(\mathbf{n p}), \\
\times\left(\frac{\operatorname{Im} A_{K, K+1}^{2}}{\sqrt{K+2}} P_{K+1}^{(2)}(\mathbf{n p})+\frac{\operatorname{Im} A_{K, K-1}^{2}}{\sqrt{K-1}} P_{K-1}^{(2)}(\mathbf{n p})\right),
\end{gathered}
$$

where the functions $P_{K}^{(j)}(x)$ are defined in (A.1) and (A.2). Note that the dynamic parameters $A_{K, K \pm 1}^{2}$ should be purely imaginary (see formula (A.6), which contains imaginary unit $i$ on its right-hand side).

Expressions (18) for the functions $a_{K}^{(3)}$ and $a_{K}^{(4)}$ are valid at $K>1$. At $K=1$, it is necessary to discard in these formulas the terms with the dynamic parameter $A_{10}^{2}$, which cannot appear according to the triangle condition $\Delta(K, l, r)$ for bipolar harmonic (13). In addition, at $K=1$, the function $a_{1}^{(3)}=0$ (see (18)); therefore, the corresponding term in (17) is absent. However, this is obvious: the third term in (17) is at least quadratic in $\mathbf{n}$ and thus cannot appear in the expression for the photoionization cross section of the atom oriented in the first order (see expression (3) for the density matrix).

\section{Even $K$ (Alignment)}

Using formulas (A.3), (A.4), and (A.8) for bipolar harmonics; definition (10) of tensors $T_{r}$; and identities (9) and (15), we can obtain from (11) and (12) the following expression for the quantities of interest:

$$
\begin{gathered}
d \sigma_{K}(\mathbf{n}) / d \Omega=b_{K}^{(0)}+b_{K}^{(1)} \xi(\mathbf{k}[\mathbf{n}, \mathbf{p}]) \\
+b_{K}^{(2)}\left(1-3|\mathbf{n e}|^{2}\right)+b_{K}^{(3)}\left(1-3|\mathbf{p e}|^{2}\right) \\
+b_{K}^{(4)}\left\{3 \operatorname{Re}\left[(\mathbf{n e})\left(\mathbf{p} \mathbf{e}^{*}\right)-(\mathbf{n p})\right]\right\} .
\end{gathered}
$$


The scalar functions $b_{K}^{(j)}(\mathbf{n p})$ are defined as follows:

$$
\begin{gathered}
b_{K}^{(0)}=\sqrt{2 K+1} A_{K K}^{0} P_{K}(\mathbf{n p}), \\
b_{K}^{(1)}=[3(2 K+1) / K(K+1)]{ }^{1 / 2} \operatorname{Im} A_{K K}^{1} P_{K}^{(1)}(\mathbf{n p}), \\
b_{K}^{(2)}=\left(D_{K, K+2}+D_{K, K-2}+D_{K}\right) P_{K}^{(2)}(\mathbf{n p}), \\
b_{K}^{(3)}=D_{K, K+2} P_{K+2}^{(2)}(\mathbf{n p}) \\
+D_{K, K-2} P_{K-2}^{(2)}(\mathbf{n p})+D_{K} P_{K}^{(2)}(\mathbf{n p}), \\
b_{K}^{(4)}=2 D_{K, K-2} P_{K-1}^{(2)}(\mathbf{n p}) \\
+2\left(D_{K, K+2}+D_{K}\right) P_{K+1}^{(2)}(\mathbf{n p})-D_{K}(2 K+3) P_{K}^{(1)}(\mathbf{n p}),
\end{gathered}
$$

where

$$
\begin{aligned}
D_{k, q} & =A_{k q}^{2}\left[(K+q+1)\left(\frac{K+q}{2}\right) \max (K, q)\right]^{-1 / 2}, \\
D_{K} & =A_{K K}^{2}\left[\frac{6(2 K+1)}{(2 K-1)(2 K+3) K(K+1)}\right]^{1 / 2},
\end{aligned}
$$

with the parameters $A_{K K}^{1}$ being purely imaginary (see (A.4)).

Formulas (6) and (17)-(20) represent the results obtained in [17] in a somewhat different form convenient for further generalizations. The dynamic parameters $A_{K l}^{r}$ introduced here are connected with the coefficients $B_{l K}^{r}$ of [17] by the relation

$$
A_{K l}^{r}=4 \pi^{2} \alpha \omega(-1)^{K-r} B_{l K}^{r},
$$

where $\omega$ is the frequency of the electromagnetic radiation and $\alpha$ is the fine structure constant. The dynamic parameters depend on the type of a system subjected to ionization and on approximations used in atomic calculations. In the case of photoionization of atoms (but not molecules), the explicit expressions for these parameters were obtained in [8] (see also [17]). As was noted in the preceding section, if the rank $K$ is odd (orientation), the state multipoles $\rho_{K}^{n}$ are $T$-odd pseudoscalars; therefore, all the terms in (17) should have the same properties with respect to spatial inversion and time reversal (the cross section (6) should be $T$-even scalar). The pseudoscalarity of all the terms in (17) is obvious. At the same time, under the operation of time reversal, which reverses the directions of the vectors $\mathbf{p}$ and $\mathbf{k}$ and replaces the vector $\mathbf{e}$ with the vector $\mathbf{e}^{*}$, the vector combinations in the first and second terms change their sign, while those in the third and fourth terms remain unchanged ( $a_{K}^{(2)}$ and $a_{K}^{(3)}$ are odd functions of the scalar product (np)). Consequently, the dynamic parameters $A_{K, K \pm 1}^{2}$ (see (18)) should be $T$-odd. Under the standard approximation, the $T$ oddness arises as a result of the proportionality of these parameters to the sine of the scattering phase difference of a photoelectron ${ }^{4}$ [17]. Therefore, at large energies of a photoelectron, at which the Born approximation holds, the $T$-odd parameters go to zero and the third and fourth terms in (17) vanish.

Note that the width of the quasi-stationary level is also $T$-odd. This $T$-odd parameter manifests itself in the scattering of light by unpolarized [21], oriented [18, 22], and aligned [23] atoms. However, simple physical considerations show that photoionization via an autoionization resonance cannot lead to the appearance of the third and fourth terms in (17). Indeed, the probability of excitation of an autoionization state upon absorption of a photon by a polarized atom should depend on the vectors $\mathbf{e}$ and $\mathbf{n}$ but not on the vector $\mathbf{p}$ determining the direction of motion of a photoelectron.

The even-rank state multipoles $\rho_{K}^{n}$ are $T$ - and $P$-even; hence, all the terms in (19) should possess the same symmetry properties. At the same time, the second term contains a $T$-odd combination of vectors $\left(b_{K}^{(1)}\right.$ is an odd function of the variable (np)); therefore, the dynamic parameter $A_{K K}^{1}$ should also be $T$-odd. This parameter, as well as the second term in (19), vanishes in the Born approximation.

In conclusion to this section, one more note should be made concerning the performance of the complete experiment. Different bipolar harmonics (13) obviously lead to the appearance of linearly independent scalars in the expression for the photoionization cross section; therefore, the coefficients of these scalars can be found from experimental measurements. The number of different bipolar harmonics and of the corresponding coefficients, the dynamic parameters $A_{K l}^{r}$, proves to be as follows (see (14), (17)-(20)).

For $K=0$, there are two parameters $A_{00}^{0}$ and $A_{02}^{0}$ directly related (see (16)) with the total photoionization cross section $\sigma$ of unpolarized atoms and the angular asymmetry parameter $\beta$. If $K=1$ (first-order orientation), there are three parameters: $A_{10}^{1}, A_{12}^{1}$, and $A_{12}^{2}$. For odd $K \geq 3$ (orientation of higher orders), there are four parameters: $A_{K, K \pm 1}^{1}$ and $A_{K, K \pm 1}^{2}$. Finally, for even $K$ (alignment), there exist five parameters: $A_{K K}^{0}, A_{K K}^{1}$, $A_{K K}^{2}$, and $A_{K, K \pm 2}^{2}$.

At $K \geq 1$, the dynamic parameters are multiplied by the corresponding state multipole and are present in the cross section as $\rho_{K}^{n} A_{K l}^{r}$; therefore, to find the dynamic parameters, knowledge of the polarization state of the atom is necessary. For given $K$, the ratio of the experi-

\footnotetext{
${ }^{4}$ The $T$-odd parameter also contributes to the spin polarization of a photoelectron [3].
} 
mentally determined coefficients is independent of $\rho_{K}^{n}$. Therefore, it is possible to find the ratios $A_{K l}^{r} / A_{K, K-1}^{1}$ (two quantities at $K=1$ and three quantities at $K=3,5$, $7, \ldots$ ) and $A_{K l}^{r} / A_{K K}^{0}$ (four quantities at $K=2,4,6, \ldots$ ) from the results of experimental measurements without knowledge of the polarization state of the system subjected to ionization.

\section{PHOTOIONIZATION OF POLARIZED OPTICALLY ACTIVE MOLECULES}

Optically active (chiral) molecules are characterized not only by scalar but also by pseudoscalar ( $P$-odd, i.e., changing their sign under the operation of spatial inversion) dynamic parameters. Under the operation of spatial inversion, the pseudoscalar parameter that characterizes the dextro(levo)rotatory isomer of a chiral molecule changes to the corresponding parameter of the levo(dextro)rotatory isomer of this molecule.

The photoionization cross section of an optically active molecule should contain additional terms. Indeed, decomposition (12) of irreducible tensors $A_{r}(\mathbf{n}, \mathbf{p})$ in terms of bipolar harmonics will now contain bipolar harmonics with both even and odd internal ranks $l$. The odd-rank bipolar harmonics in (12) are constructed from the $P$-odd spherical function $Y_{I M}(\mathbf{p})$ and are multiplied by the pseudoscalar dynamic parameter $A_{K l}^{r}$ ( $l$ is odd) of the chiral molecule. In atoms and optically inactive molecules, $A_{K l}^{r}=0$ for odd $l$. From the above, using the results of Section 3, it is easy to determine the dependence of the photoionization cross section (6) on the geometric parameters.

In the angular distribution of photoelectrons escaping from unpolarized chiral molecules, an additional term appears containing the pseudoscalar parameter $A_{01}^{1}$. By repeating the calculations that resulted in (14) and applying either formula (A.5) or simply the definitions of the bipolar harmonic and spherical function, we can write the sought angular distribution as

$$
\frac{d \sigma}{d \Omega}=\frac{\sigma}{4 \pi}\left[1+\beta \frac{3|\mathbf{e p}|^{2}-1}{2}+\beta_{a} \xi(\mathbf{k p})\right] .
$$

Here, the relation of the total cross section $\sigma$ and the angular-asymmetry scalar parameter $\beta$ with the dynamic parameters $A_{00}^{0}$ and $A_{02}^{0}$ was already found (see (16)) and the angular asymmetry pseudoscalar parameter is given by

$$
\beta_{a}=\sqrt{3} A_{01}^{1} / A_{00}^{0} .
$$

The last term in (21), which vanishes upon integration over all the directions of motion of the photoelectron, determines the effect of circular dichroism in the angular distribution of photoelectrons (CDADP). The effects of spin polarization of photoelectrons formed upon photoionization of unpolarized chiral molecules were discussed by Cherepkov [3].

To determine the dependence of the quantities $d \sigma_{K} / d \Omega$ present in (6) on the geometric parameters, it suffices to apply in a certain fashion formulas (17)-(20) of the preceding section.

\section{Odd $K$ (Orientation)}

Additional terms on the right-hand side of (17) appear due to bipolar harmonics containing $Y_{I M}(\mathbf{p})$ with odd $l$ in decomposition (12). Therefore, as follows from formulas (A.3), (A.4), (A.7), and (A.8) reducing bipolar harmonics, these terms should be determined by the right-hand side of (19), in which the pseudoscalar functions $\rho_{K}^{n} Y_{K Q}$ (n) ( $K$ is odd) differ only in sign from the functions defined in (20). The difference in sign is due to the fact that the above-listed formulas reducing bipolar harmonics contain the factor $(-1)^{K}$. Therefore, to find $d \sigma_{K} / d \Omega$, it is necessary to subtract from the righthand side of (17) the right-hand side of (19) with the functions $b_{K}^{(j)}$ defined in (20). The pseudoscalars $b_{K}^{(j)}$ are expressed via the pseudoscalar dynamic parameters of the optically active molecule $A_{K l}^{r}$ with odd $l$.

At $K=1$ (first-order orientation), the term with the coefficient $D_{1,-1}$ in (20), which formally contains the parameter $A_{1,-1}^{2}$, should be discarded; the corresponding bipolar harmonic simply does not exist (see (13) at $l=-1)$. In addition, at $K=1$, the function $b_{1}^{(2)}=0$ and the corresponding term in (19) is absent, which is quite natural: due to the first-order orientation, the expression for the photoionization cross section should contain only terms proportional to $\mathbf{n}$.

\section{Even K (Alignment)}

Considerations similar to those given above show that, in order to find $d \sigma_{K} / d \Omega$ in this case, it is necessary to subtract the right-hand side of (17) from the righthand side of (19) (see formulas (A.5) and (A.6) reducing bipolar harmonics). The pseudoscalar functions $a_{K}^{(j)}(\mathbf{n p})$ defined by formulas (18) with even $K$ are linear in the dynamic pseudoscalars $A_{K l}^{r}$ ( $l$ is odd) of an optically active molecule.

Note also that the pseudoscalars $A_{K, K \pm 1}^{2}$ ( $K$ is even) and $A_{K K}^{1}$ ( $K$ is odd) are $T$-odd and, as was discussed in Section 3, vanish in the Born approximation.

In the case of an optically active molecule, the general number of dynamic parameters that can be determined from experimental measurements increases (see the discussion at the end of the preceding section). At 
$K=0$, there are three parameters, $A_{00}^{0}, A_{02}^{2}$, and $A_{01}^{1}$, which are related to $\sigma, \beta$, and $\beta_{a}$. At $K=1$, four additional pseudoscalar parameters appear, $A_{11}^{0}, A_{11}^{1}, A_{11}^{2}$, and $A_{13}^{2}$, so that there are seven parameters altogether. At odd $K \geq 3$, we have five additional parameters, $A_{K K}^{0}$, $A_{K K}^{1}, A_{K K}^{2}$, and $A_{K, K \pm 2}^{2}$, and nine parameters in all. Finally, at even $K$, there are four additional parameters $A_{K, K \pm 1}^{1}, A_{K, K \pm 1}^{2}$ and nine parameters altogether.

\section{THE TOTAL PHOTOIONIZATION CROSS SECTION}

The dependence of the total photoionization cross section of an axisymmetrically polarized atom on the geometric parameters can be determined from the symmetry considerations, without addressing the differential cross section (6). Indeed, by analogy with (11), we can write the total cross section in the form

$$
\sigma(\mathbf{n})=\sum_{r=0}^{2}\left(A_{r}(\mathbf{n}) T_{r}\right),
$$

where the irreducible $r$-rank tensors $T_{r}$ composed of the polarization vector $\mathbf{e}$ are defined in (10), while the irreducible tensors $A_{r m}(\mathbf{n})$ should be proportional to the spherical functions $Y_{r m}(\mathbf{n})$. Based on the general structure of the transition probability (4) and density matrix of the polarized atom (3), we obtain that

$$
A_{1}(\mathbf{n})=\gamma_{1} \rho_{1}^{n} \mathbf{n}, \quad A_{2}(\mathbf{n})=\sqrt{5} \gamma_{2} \rho_{2}^{n}\{\mathbf{n} \otimes \mathbf{n}\}_{2},
$$

where $\gamma_{1}$ and $\gamma_{2}$ are scalar dynamic parameters, while the scalar $A_{0}$ coincides with the total photoionization cross section $\sigma$ of the unpolarized system. Substituting these expressions for $A_{r}(\mathbf{n})$ into (22) and taking into account the definition (10) of the tensors $T_{r}$ and identities (9) and (15), we obtain the following general expression for the total photoionization cross section of a polarized atomic-molecular system:

$$
\sigma(\mathbf{n})=\sigma+\rho_{1}^{n} \gamma_{1} \xi(\mathbf{k n})+\rho_{2}^{n} \gamma_{2}\left(1-3|\mathbf{n e}|^{2}\right) .
$$

Therefore, the expression for the total photoionization cross section can contain only the first-order orientation and alignment.

It is underlined once more that the structure of the expression for the total photoionization cross section of chiral molecules is the same (23) as that for atoms and optically inactive molecules. This means that all additional chiral terms present in the expression for the differential photoionization cross section of an optically active molecule vanish after integration over the directions of motion of a photoelectron. The symmetry considerations used in substantiation of (23) clearly point to the reason why chiral effects do not manifest them- selves in the expression for the total photoionization cross section: no pseudoscalar can be composed of the $P$-even irreducible tensors $\rho_{r}^{n} Y_{r m}(\mathbf{n})$ and $T_{r m}$; therefore, no pseudoscalar dynamic parameters of an optically active molecule can appear in the expression for the total cross section of the process (as was noted above, the parameters $\gamma_{1}$ and $\gamma_{2}$ are scalars). For the same reason, chiral effects do not manifest themselves in the processes of dipole emission and absorption and scattering of light by optically active molecules.

The coefficients $\gamma_{1}$ and $\gamma_{2}$ in (23) can be associated with the dynamic parameters $A_{K l}^{r}$. For this, it suffices, by integrating the angular distribution (6) over all the directions $\mathbf{p}$ of motion of a photoelectron, to obtain by another method the expression determining the photoeffect total cross section. The integration is easy to perform if one notices that the dependence on the vector $\mathbf{p}$ in (6) arises because the expressions for the bipolar harmonics contain the spherical functions $Y_{l m}(\mathbf{p})$ (see (11) and (12)). At the same time,

$$
\int Y_{l m}(\mathbf{p}) d \Omega=0,
$$

if $l \neq 0$. Therefore, after the integration, only terms containing the dynamic parameters $A_{00}^{0}$ (the contribution of the unpolarized system), $A_{10}^{1}$ (the contribution of the first-order orientation), and $A_{20}^{2}$ (the contribution of the first-order alignment) will remain in the expression for the total cross section. It is clear that, in the case of an optically active molecule, all the chiral terms (the terms proportional to the spherical functions of odd rank $l$ ) vanish after integration. By gathering the terms in (14) (or in (7)), (17), and (19) containing these dynamic parameters (such terms are independent of $\mathbf{p}$ ) and performing elementary integration (which reduces to multiplying by $4 \pi$ ), we obtain the expression for the total photoionization cross section in the form of (23), where

$$
\gamma_{1}=\sqrt{3} A_{10}^{1}, \quad \gamma_{2}=\sqrt{3 / 2} A_{20}^{2} \text {. }
$$

The relation between $\sigma$ and $A_{00}^{0}$ was already given above (see (16)).

\section{PARTIAL POLARIZATION OF IONIZING RADIATION}

It was assumed in the preceding sections that electromagnetic radiation ionizing an atom is completely polarized, so that its polarization can be specified by the vector e. In the more general case of a partial polarization, the polarization state of a photon is determined by three polarization parameters, for example, the Stokes parameters $\eta_{i}, i=1,2,3[11,24]$. The parameter $\eta_{2}$ determines the degree of circular polarization, the parameter $\eta_{3}$ characterizes the degree of linear polar- 
ization along the axes $x$ and $y$ (the axis $z$ is oriented along the direction of propagation of the light wave), and the parameter $\eta_{1}$ determines the degree of linear polarization along the axes $p$ and $q$ that are rotated in the positive direction by an angle of $45^{\circ}$ in the $x y$ plane with respect to the axes $x$ and $y$. The Stokes parameters satisfy the relation

$$
\eta_{1}^{2}+\eta_{2}^{2}+\eta_{3}^{2} \leq 1
$$

with the equality sign corresponding to the complete polarization. For unpolarized light, all the three Stokes parameters go to zero.

As was shown in [25], the cross section of any photoprocess accompanied by either absorption or induced emission of a partially polarized photon can be expressed in terms of the Stokes parameters and three dichroism terms of this process,

$$
\frac{d \sigma}{d \Omega}=\frac{d \sigma_{0}}{d \Omega}+\frac{1}{2}\left(\eta_{1} \frac{d \sigma_{p q}}{d \Omega}+\eta_{2} \frac{d \sigma_{+-}}{d \Omega}+\eta_{3} \frac{d \sigma_{x y}}{d \Omega}\right) .
$$

Here, $d \sigma_{0} / d \Omega$ is the photoprocess cross section with the participation of an unpolarized photon; $d \sigma_{+-} / d \Omega$ is the difference between the cross sections for the cases of the right- and left-hand circular polarizations, specified by the vector $\mathbf{e}_{ \pm}$(the circular dichroism of the process); and $d \sigma_{x y} / d \Omega$ is the difference between the cross sections for the cases of linear polarizations along the axes $x$ and $y$ (the linear $x y$ dichroism). The corresponding vectors of the linear polarizations will be denoted as $\mathbf{e}_{x}$ and $\mathbf{e}_{y}$. Finally, $d \sigma_{p q} / d \Omega$ is the linear $p q$ dichroism of this process. Formula (24) makes it possible to separate comparatively simply the dependence on the Stokes parameters in the expression for the photoprocess cross section; as a result, this expression becomes applicable in the general case of a partial polarization of electromagnetic radiation.

The passage to unpolarized light is reduced to the averaging with the statistical weight $1 / 2$ of all the expressions for the photoionization cross section obtained above over two orthogonal polarization vectors $\mathbf{e}_{\lambda}$. In this case, the terms with the degree of circular polarization $\xi$ vanish and the remaining terms containing the polarization vector e can be easily averaged with the help of the identity [26]

$$
\sum_{\lambda}\left(\mathbf{a e}_{\lambda}\right)\left(\mathbf{b} \mathbf{e}_{\lambda}^{*}\right)=([\mathbf{k}, \mathbf{a}][\mathbf{k}, \mathbf{b}]) .
$$

The term for linear dichroism is found by simple substitution of the corresponding real-valued vectors of linear polarizations into the expression for the photoionization cross section. To find the term for circular dichroism, in all the terms, the parameter $\xi$, equal to \pm 1 for the right-hand and left-hand polarizations, respectively, is replaced with 2 . As a result, according to (24), these terms will be present in the final expression virtually unchanged and only the parameter $\xi$ will be changed to $\eta_{2} \cdot{ }^{5}$ All the remaining terms do not contribute to the circular dichroism, which can be easily shown with the help of the identity

$$
\left(\mathbf{a e}_{ \pm}\right)\left(\mathbf{b e}_{ \pm}^{*}\right)=\{([\mathbf{k}, \mathbf{a}][\mathbf{k}, \mathbf{b}]) \pm i(\mathbf{k}[\mathbf{b}, \mathbf{a}])\} / 2 .
$$

After these remarks, we can easily obtain the expressions for the quantities $d \sigma / d \Omega$ and $d \sigma_{K} / d \Omega$, which determine the angular distribution of photoelectrons (6) and the total photoionization cross section in the general case of a partial polarization of electromagnetic radiation ionizing an atom (molecule).

By going to the partial polarization of light in formulas (7) and (21) according to rule (24), we obtain the following expression for the angular distribution of photoelectrons escaping from unpolarized atoms (molecules):

$$
\begin{aligned}
& \frac{d \sigma}{d \Omega}=\frac{\sigma}{4 \pi}\left[1+\beta \frac{3[\mathbf{k}, \mathbf{p}]^{2}-2}{4}\right. \\
& \left.+\beta_{a} \eta_{2}(\mathbf{k p})+\eta_{1} \delta_{p q}+\eta_{3} \delta_{x y}\right],
\end{aligned}
$$

where

$$
\delta_{i j}=(3 \beta / 4)\left[\left(\mathbf{e}_{i} \mathbf{p}\right)^{2}-\left(\mathbf{e}_{j} \mathbf{p}\right)^{2}\right] .
$$

Here, the angular-asymmetry pseudoscalar parameter $\beta_{a}$ differs from zero only in the case of photoionization of optically active molecules.

Then, we pass to the partial polarization of electromagnetic radiation in formulas (17) and (19) and obtain expressions for the quantities $d \sigma_{K} / d \Omega$, which enter the angular distribution of photoelectrons escaping from polarized atoms and achiral molecules.

\section{Odd-Rank K State Multipoles (Orientation)}

In this case, we have

$$
\begin{gathered}
d \sigma_{K}(\mathbf{n}) / d \Omega=(1 / 2) a_{K}^{(3)}(\mathbf{k}[\mathbf{n}, \mathbf{p}])(\mathbf{k n}) \\
+(1 / 2) a_{K}^{(4)}(\mathbf{k}[\mathbf{p}, \mathbf{n}])(\mathbf{k p})+a_{K}^{(1)} \eta_{2}(\mathbf{k n}) \\
+a_{K}^{(2)} \eta_{2}(\mathbf{k p})+\eta_{1} \delta_{K, p q}+\eta_{3} \delta_{K, x y}
\end{gathered}
$$

where

$$
\begin{gathered}
\delta_{K, i j}=\left(\left\{a_{K}^{(3)}\left[\mathbf{e}_{i}\left(\mathbf{n} \mathbf{e}_{i}\right)-\mathbf{e}_{j}\left(\mathbf{n} \mathbf{e}_{j}\right)\right]\right.\right. \\
\left.\left.-a_{K}^{(4)}\left[\mathbf{e}_{i}\left(\mathbf{p} \mathbf{e}_{i}\right)-\mathbf{e}_{j}\left(\mathbf{p} \mathbf{e}_{j}\right)\right]\right\}[\mathbf{p}, \mathbf{n}]\right) / 2,
\end{gathered}
$$

and the scalar functions $a_{K}^{(j)}(\mathbf{n p})$ are defined in (18).

\footnotetext{
${ }^{5}$ According to the definition, $\xi=\eta_{2}$ in the case of a completely polarized electromagnetic wave.
} 
Even-Rank K State Multipoles (Alignment)

Here,

$$
\begin{gathered}
\frac{d \sigma_{K}(\mathbf{n})}{d \Omega}=b_{K}^{(0)}+(1 / 2) b_{K}^{(2)}\left[3(\mathbf{k n})^{2}-1\right] \\
+(1 / 2) b_{K}^{(3)}\left[3(\mathbf{k p})^{2}-1\right] \\
+(1 / 2) b_{K}^{(4)}[(\mathbf{n p})-3(\mathbf{k n})(\mathbf{k p})] \\
+b_{K}^{(1)} \eta_{2}(\mathbf{k}[\mathbf{n}, \mathbf{p}])+\eta_{1} \delta_{K, p q}+\eta_{3} \delta_{K, x y},
\end{gathered}
$$

where

$$
\begin{gathered}
\delta_{K, i j}=\left\{b_{K}^{(2)}\left[\left(\mathbf{n} \mathbf{e}_{j}\right)^{2}-\left(\mathbf{n} \mathbf{e}_{i}\right)^{2}\right]\right. \\
+b_{K}^{(3)}\left[\left(\mathbf{p} \mathbf{e}_{j}\right)^{2}-\left(\mathbf{p} \mathbf{e}_{i}\right)^{2}\right] \\
\left.+b_{K}^{(4)}\left[\left(\mathbf{n} \mathbf{e}_{i}\right)\left(\mathbf{p} \mathbf{e}_{i}\right)-\left(\mathbf{n} \mathbf{e}_{j}\right)\left(\mathbf{p} \mathbf{e}_{j}\right)\right]\right\} 3 / 2,
\end{gathered}
$$

and the scalar functions $b_{K}^{(j)}(\mathbf{n p})$ are defined in (20).

In the case of optically active molecules, as follows from the results of Section 5, the expressions for $d \sigma_{K} / d \Omega$ in (6) can be obtained by combining formulas (27) and (28). In the case of odd-rank $K$ state multipoles, $d \sigma_{K} / d \Omega$ is determined by the difference between the right-hand sides of (27) and (28) and the functions $b_{K}^{(j)}(\mathbf{n p})$ defined in (20) are pseudoscalars, which are expressed via the pseudoscalar dynamic parameters of the chiral molecule. For even-rank $K$ state multipoles, $d \sigma_{K} / d \Omega$ can be found by subtracting the right-hand side of (27) from the right-hand side of (28). In this case, the functions $a_{K}^{(j)}(\mathbf{n p})$ defined in (18) are pseudoscalars.

Expression (23) for the total photoionization cross section can also be easily transformed to the general case of the partial polarization of an electromagnetic wave,

$$
\begin{gathered}
\sigma(\mathbf{n})=\sigma+\rho_{1}^{n} \gamma_{1} \eta_{2}(\mathbf{k n}) \\
+(1 / 2) \rho_{2}^{n} \gamma_{2}\left[3(\mathbf{k n})^{2}-1\right]+\eta_{1} \Delta_{p q}+\eta_{3} \Delta_{x y},
\end{gathered}
$$

where

$$
\Delta_{i j}=(3 / 2) \rho_{2}^{n} \gamma_{2}\left[\left(\mathbf{n e}_{j}\right)^{2}-\left(\mathbf{n e}_{i}\right)^{2}\right] .
$$

Another form of the expression for the photoprocess cross section with the participation of a partially polarized photon, which was briefly discussed in [25], can be obtained by a special selection of the coordinate system. Let $\tilde{x}$, and $\tilde{y}$ be the axes of a coordinate system, in which the Stokes parameter $\tilde{\eta}_{1}=0$ and which is rotated by some angle in the plane perpendicular to the direction of propagation of a wave. The rotation corresponds to the diagonalization of the real-valued part of the polarization density matrix of a photon defined in a Cartesian basis [24], and the Stokes parameter

$$
\tilde{\eta}_{3}=\sqrt{\eta_{1}^{2}+\eta_{3}^{2}}=l
$$

is termed the degree of linear polarization of the wave, while the angle of rotation $\varphi$ is defined by the conditions

$$
\cos 2 \varphi=\eta_{3} / l, \quad \sin 2 \varphi=\eta_{1} / l .
$$

The state of the partial polarization of the photon is now specified by the following three parameters: $l, \varphi$, and $\eta_{2}$. Further, we will denote the real-valued basis vector $\mathbf{e}_{x}$ by $\mathbf{g}$ and say that the vector $\mathbf{g}$ is directed along the principal axis of the linear polarization (for each specified polarization state of the wave, the degree of linear polarization along the axes $\tilde{x}$ and $\tilde{y}$ is maximal possible and equal to $l$ ). It is easy to show that, in the case of the complete polarization of light, defined by the vector $\mathbf{e}\left(\eta_{2}^{2}+l^{2}=1\right)$, the $\tilde{x}$ and $\tilde{y}$ axes coincide with the axes of the polarization ellipse, while the vector $\mathbf{g}$ is directed along the major axis of this ellipse.

The form of the expression for the photoprocess cross section in the coordinate system $(\tilde{x}, \tilde{y})$ will be termed the $(\mathbf{g}, l)$ form. To obtain this form, it suffices to set in (24) $\eta_{1}=\tilde{\eta}_{1}=0$ and $\eta_{3}=\tilde{\eta}_{3}=l$ and to express the term for linear $\tilde{x} \tilde{y}$ dichroism via the photoprocess cross section $d \sigma_{\mathrm{g}} / d \Omega$ with the participation of a photon polarized linearly along the principal axis of the linear polarization $\mathbf{g}$ and the photoprocess cross section $d \sigma_{0} / d \Omega$ with the participation of an unpolarized photon,

$$
\begin{gathered}
d \sigma_{\tilde{x} \tilde{y}} / d \Omega=d \sigma_{\mathbf{e}_{\tilde{x}}} / d \Omega-d \sigma_{\mathbf{e}_{\tilde{y}}} / d \Omega \\
=2 d \sigma_{\mathbf{g}} / d \Omega-2 d \sigma_{0} / d \Omega .
\end{gathered}
$$

Therefore, the $(\mathrm{g}, l)$ form of the cross section is given by

$$
\begin{aligned}
d \sigma / d \Omega & =(1-l) d \sigma_{0} / d \Omega+l d \sigma_{\mathrm{g}} / d \Omega \\
& +(1 / 2) \eta_{2} d \sigma_{+-} / d \Omega .
\end{aligned}
$$

Here, for the linear polarization along $\mathbf{g}, \eta_{2}=0$ and $l=1$; for the left-hand (right-hand) circular polarization, $l=0$ and $\eta_{2}= \pm 1$; and, for the unpolarized light, $l=\eta_{2}=0$. Note that, in [17], the dependence of the cross section on $l$ and $\mathbf{g}$ was artificially separated for the case of the complete polarization and termed a new convenient parameterization of the polarization state of a wave. As follows from the above, (g, $l$ ) form (30) should be considered as a partial case of the expression for the cross section in terms of the Stokes parameters in a coordinate system oriented in a particular way. Thus, in a previous study [27], the dependence of the cross section of two-electron photoionization on the Stokes parameters was presented in precisely this form.

The simplest way to express formulas (26)-(29) in the $(\mathbf{g}, l)$ form without using (30) is to set $\eta_{1}=0$ and 
$\eta_{3}=l$ in these formulas and to apply the identity (see (25))

$$
\begin{gathered}
\left(\mathbf{e}_{\tilde{x}} \mathbf{a}\right)\left(\mathbf{e}_{\tilde{x}} \mathbf{b}\right)-\left(\mathbf{e}_{\tilde{y}} \mathbf{a}\right)\left(\mathbf{e}_{\tilde{y}} \mathbf{b}\right) \\
=2(\mathbf{g a})(\mathbf{g b})-([\mathbf{k}, \mathbf{a}][\mathbf{k}, \mathbf{b}]) .
\end{gathered}
$$

The final expressions are as follows:

$$
\begin{gathered}
\frac{d \sigma}{d \Omega}=\frac{\sigma}{4 \pi}\left[1+\beta \frac{3(1-l)[\mathbf{k}, \mathbf{p}]^{2}-2}{4}\right. \\
\left.+\frac{3}{2} \beta l(\mathbf{g p})^{2}+\beta_{a} \eta_{2}(\mathbf{k p})\right], \\
d \sigma_{K}(\mathbf{n}) / d \Omega=(1 / 2) a_{K}^{(3)}(1-l)(\mathbf{k}[\mathbf{n}, \mathbf{p}])(\mathbf{k n}) \\
+(1 / 2) a_{K}^{(4)}(1-l)(\mathbf{k}[\mathbf{p}, \mathbf{n}])(\mathbf{k p})+a_{K}^{(1)} \eta_{2}(\mathbf{k n}) \\
+a_{K}^{(2)} \eta_{2}(\mathbf{k p})+a_{K}^{(3)} l(\mathbf{g}[\mathbf{p}, \mathbf{n}])(\mathbf{g n}) \\
+a_{K}^{(4)} l(\mathbf{g}[\mathbf{n}, \mathbf{p}])(\mathbf{g p})
\end{gathered}
$$

for odd $K$ and

$$
\begin{gathered}
d \sigma_{K}(\mathbf{n}) / d \Omega \\
=b_{K}^{(0)}+(1 / 2) b_{K}^{(2)}\left[3(1-l)(\mathbf{k n})^{2}+3 l-1\right] \\
+(1 / 2) b_{K}^{(3)}\left[3(1-l)(\mathbf{k p})^{2}+3 l-1\right] \\
+(1 / 2) b_{K}^{(4)}[(1-3 l)(\mathbf{n p})-3(1-l)(\mathbf{k n})(\mathbf{k p})] \\
+b_{K}^{(1)} \eta_{2}(\mathbf{k}[\mathbf{n}, \mathbf{p}])-3 b_{K}^{(2)} l(\mathbf{n g})^{2}-3 b_{K}^{(3)} l(\mathbf{p g})^{2} \\
+3 b_{K}^{(4)} l(\mathbf{n g})(\mathbf{p g})
\end{gathered}
$$

for even $K$,

$$
\begin{gathered}
\sigma(\mathbf{n})=\sigma+\rho_{1}^{n} \gamma_{1} \eta_{2}(\mathbf{k n}) \\
+(1 / 2) \rho_{2}^{n} \gamma_{2}\left[3(1-l)(\mathbf{k n})^{2}+3 l-1\right] \\
-3 \rho_{2}^{n} \gamma_{2} l(\mathbf{n g})^{2} .
\end{gathered}
$$

Expressions (31) and (32) determine the quantities $d \sigma_{K} / d \Omega$ present in the angular distribution of photoelectrons (6) escaping from polarized atoms and polarized optically inactive molecules. In the case of optically active molecules, the expressions for these quantities are composed of (31) and (32), as was shown above.

Note that the second term in expressions (23), (29), and (33) for the complete cross section, which is related to the orientation, depends on the circular polarization of light and, therefore, determines the effect of circular dichroism in the photoionization process [13], whereas the dependence of the total cross section on the degree of linear polarization of light arises due to the alignment.

\section{PHOTOIONIZATION \\ OF AN ORIENTED SYSTEM}

In this section, we will analyze in more detail the influence of the effects of the orientation of an atom or molecule on the angular distribution of photoelectrons. We will restrict ourselves to the simplest case of firstorder orientation. It should be kept in mind that, at $j=$ $1 / 2$, at which the system is characterized by only one state multipole of nonzero rank $\rho_{1 Q}$, the polarization of the system is equivalent to its first-order orientation. If $j>1 / 2$, the first-order orientation is a particular type of polarization at which the state multipoles of the second and higher ranks are equal to zero. Note also that, for first-order orientation, all the formulas obtained for the axisymmetric polarization retain their form in the general case of an asymmetrically polarized system if $\mathbf{n}$ is taken to mean the unit vector collinear to the average angular momentum $\overline{\mathbf{j}}$ of the polarized atom. Indeed, as follows from formula (2), in a coordinate system whose $z$ axis is directed along $\mathbf{n}, \rho_{1, \pm 1}=0$ and only one component of the state multipole, $\rho_{10}=\rho_{1}^{n}$, is nonzero.

The structure of the expression for the angular distribution of photoelectrons is the same as that of (6), where the first term (see (7), (21), and (26)) characterizes the angular distribution of photoelectrons formed upon photoionization of an unpolarized system, while the contribution of the effects of the first-order orientation is determined by the quantity $d \sigma_{1} / d \Omega$. As was shown in Sections 3, 4, and 6, to find this quantity, it suffices to represent in the explicit form the scalar functions $a_{1}^{(j)}(\mathbf{n p})$ given by (18) and, in the case of optically active molecules, the pseudoscalar functions $b_{1}^{(j)}(\mathbf{n p})$ from (20) as well. To write the result in a more compact form, we will introduce scalars proportional to the scalar dynamic parameters $A_{1 l}^{r}$,

$$
\Gamma=\sqrt{3} A_{10}^{1}, \quad \Gamma_{1}=-\sqrt{3 / 2} A_{12}^{1}, \quad \Gamma_{2}=-3 \sqrt{3} \operatorname{Im} A_{12}^{2},
$$

and pseudoscalars related to the pseudoscalar dynamic parameters of an optically active molecule,

$$
\begin{gathered}
v=(\sqrt{6 / 5}-\sqrt{15 / 2}) A_{13}^{2}+(3 / \sqrt{5}) A_{11}^{2}-\sqrt{3} A_{11}^{0}, \\
v_{1}=(-3 / \sqrt{2}) \operatorname{Im} A_{11}^{1}, \\
v_{2}=(-3 / \sqrt{5})\left(\sqrt{6} A_{13}^{2}+3 A_{11}^{2}\right), \quad v_{3}=3 \sqrt{15 / 2} A_{13}^{2} .
\end{gathered}
$$

In accordance with definitions (18), (20), (A.1), and (A.2), we have

$$
\begin{gathered}
a_{1}^{(1)}=\Gamma-\Gamma_{1}, \quad a_{1}^{(2)}=3 \Gamma_{1}(\mathbf{n p}), \quad a_{1}^{(3)}=0, \\
a_{1}^{(4)}=-\Gamma_{2}, \\
b_{1}^{(0)}=-\left(v+v_{2} / 3+v_{3} / 3\right)(\mathbf{n p}), \quad b_{1}^{(1)}=-v_{1}, \\
b_{1}^{(2)}=0, \quad b_{1}^{(3)}=v_{3}(\mathbf{n p}) / 3, \quad b_{1}^{(4)}=-v_{2} / 3 .
\end{gathered}
$$


Then, as was described in Section 4, by subtracting the right-hand side of (19) from the right-hand side of (17), we obtain the expression for $d \sigma_{1} / d \Omega$ for the case where the complete polarization of ionizing radiation is defined by the vector $\mathbf{e}$,

$$
\begin{gathered}
d \sigma_{1}(\mathbf{n}) / d \Omega=\Gamma \xi(\mathbf{k n})+\Gamma_{1} \xi[3(\mathbf{k p})(\mathbf{n p})-(\mathbf{k n})] \\
+\Gamma_{2} \operatorname{Re}\left[(\mathbf{p e})\left([\mathbf{p}, \mathbf{n}] \mathbf{e}^{*}\right)\right]+v(\mathbf{n p}) \\
+v_{1} \xi(\mathbf{k}[\mathbf{n}, \mathbf{p}])+v_{2} \operatorname{Re}\left[(\mathbf{n e})\left(\mathbf{p e} \mathbf{e}^{*}\right)\right] \\
+v_{3}(\mathbf{p n})|\mathbf{p e}|^{2}
\end{gathered}
$$

In the general case, expression (35) contains seven dynamic parameters: three scalars and four pseudoscalars (see the end of Section 4). Upon ionization of atoms and achiral molecules, the pseudoscalars $v$ and $v_{j}$ turn to zero and the number of terms in (35) decreases.

The formulas obtained in the preceding section also allow one to write the expression for $d \sigma_{1} / d \Omega$ in the more general case of a partial polarization of electromagnetic radiation. Subtracting from the right-hand side of (27) the right-hand side of (28) with the functions $a_{1}^{(j)}$ and $b_{1}^{(j)}$ from (34), we find that

$$
\begin{gathered}
d \sigma_{1}(\mathbf{n}) / d \Omega=\Gamma_{2}(\mathbf{k}[\mathbf{n}, \mathbf{p}])(\mathbf{k p}) / 2 \\
+\left(v+v_{2} / 2+v_{3} / 2\right)(\mathbf{n p})-v_{3}(\mathbf{n p})(\mathbf{k p})^{2} / 2 \\
-v_{2}(\mathbf{k n})(\mathbf{k p}) / 2+\left(\Gamma-\Gamma_{1}\right) \eta_{2}(\mathbf{k n}) \\
+3 \Gamma_{1} \eta_{2}(\mathbf{k p})(\mathbf{p n})+v_{1} \eta_{2}(\mathbf{k}[\mathbf{n}, \mathbf{p}]) \\
+\eta_{1} \delta_{1, p q}+\eta_{3} \delta_{1, x y},
\end{gathered}
$$

where

$$
\begin{aligned}
\delta_{1, i j} & =\Gamma_{2}\left(\left[\mathbf{e}_{i}\left(\mathbf{p} \mathbf{e}_{i}\right)-\mathbf{e}_{j}\left(\mathbf{p} \mathbf{e}_{j}\right)\right][\mathbf{p}, \mathbf{n}]\right) / 2 \\
+ & v_{2}\left[\left(\mathbf{n e}_{i}\right)\left(\mathbf{p} \mathbf{e}_{i}\right)-\left(\mathbf{n e}_{j}\right)\left(\mathbf{p e} \mathbf{e}_{j}\right)\right] / 2 \\
& +v_{3}(\mathbf{p n})\left[\left(\mathbf{p} \mathbf{e}_{i}\right)^{2}-\left(\mathbf{p e} \mathbf{e}_{j}\right)^{2}\right] / 2 .
\end{aligned}
$$

In a similar manner, with the help of (31) and (32) or by simple rotation of the axes, one can write $d \sigma_{1} / d \Omega$ (36) in the $(\mathbf{g}, l)$ form.

Formulas (35) and (36) make it possible to analyze various effects that can be observed in the angular distribution of photoelectrons formed upon photoionization of oriented systems. Thus, the first, second, and fifth terms in (35) (and the corresponding terms proportional to the Stokes parameter $\eta_{2}$ in (36)) determine the effect of circular dichroism in the angular distribution of photoelectrons escaping from oriented systems. In this case, the fifth term in (35) (the seventh in (36)) characterizes the CDADP effect determined by the orientation and chirality. If the direction of propagation of electromagnetic radiation $\mathbf{k}$ is perpendicular to the symmetry axis $\mathbf{n}$ of a polarized system and photoelectrons are observed in the direction $\mathbf{p}$ perpendicular to either $\mathbf{n}$ or $\mathbf{k}$, then only the orientational CDADP effect associated with the chirality is observed. It should be kept in mind that no such effect is observed for photoelectrons escaping from unpolarized optically active molecules in the direction perpendicular to $\mathbf{k}$ (see (21)); therefore, for the experimental geometry indicated above, one can observe only the CDADP effect determined simultaneously by the orientation and the chirality. Note also that the CDADP effect associated with the chirality of molecules depends on the sign of $\mathbf{p}$ (see the term in (25) and (36) proportional to $v_{1}$ ).

Recall that the parameters $\Gamma_{2}$ and $\nu_{1}$ are $T$-odd and, therefore, vanish at large energies of photoelectrons, i.e., in the Born approximation (see Section 3). Therefore, at large energies of photoelectrons, the CDADP effect determined by the orientation and optical activity vanishes, while the dependence of the angular distribution of photoelectrons on the degree of linear polarization of ionizing radiation, which is related to the orientation, is retained only due to the optical activity of molecules (36).

In conclusion to this section, it should be noted that formulas (35) and (36) have a wider domain of applicability. In particular, these formulas describe effects associated with the orientation of photoelectron spin in the photoionization process of unpolarized atomicmolecular systems. The formula for the photoionization cross section is obtained if (35) (or (36)) is added to (21) (or, correspondingly, to (26)) multiplied by $1 / 2$. In this case, the vector $\mathbf{n}$ should be replaced with the pseudovector $\mathbf{s}$, defining the direction of the spin of a photoelectron; the numerical values of the coefficients $\Gamma, \Gamma_{j}, v$, and $v_{j}$ will also change. Furthermore, if the quantum number of the total angular momentum of the residual photoion proves to be equal to $1 / 2$, the expression for the photoionization cross section of an unpolarized system taking into account the orientation of the photoion spin should have a similar structure. In particular cases of the linear and circular polarizations of electromagnetic radiation, invariant expressions for the photoionization cross section taking into account the spin polarization of photoelectrons were obtained by Cherepkov [3], and, in the general case of the complete polarization of radiation, the corresponding expression for the photoionization cross section of atoms and achiral molecules was reported in [17].

\section{PHOTOIONIZATION OF AN ALIGNED SYSTEM}

Let us consider in more detail the photoionization of systems aligned in the first order. Note that, if an external action polarizing an atom or a molecule is not only axisymmetric, but also mirror symmetric (for example, unpolarized or linearly polarized light), the magnetic sublevels with opposite projections of the angular momentum onto the symmetry axis $\mathbf{n}$ are equally populated and all odd-rank state multipoles turn to zero [11]. In this case, the system proves to be aligned, and, at $j=1$ or $1 / 2$, when only one state multipole of even 
rank $\rho_{2, Q}$ is nonzero, it is aligned in the first order. The state multipoles of the third and higher ranks are also not excited upon single-photon dipole absorption of electromagnetic radiation.

At $\rho_{2}^{n} \neq 0$, the angular distribution of photoelectrons is defined by formula (6), in which the quantity $d \sigma_{2} / d \Omega$ characterizes the structure of the addition determined by the first-order alignment. Initially, the explicit form of the scalar functions $b_{2}^{(j)}$ (np) from (20) and pseudoscalar functions $a_{2}^{(j)}(\mathbf{n p})$ from (18) present in the expression for $d \sigma_{2} / d \Omega$ will be presented. For compactness, we will introduce the five different scalar parameters

$$
\begin{gathered}
c_{0}=\sqrt{5} A_{22}^{0} / 2, \quad c_{1}=3 \sqrt{5 / 2} \operatorname{Im} A_{22}^{1}, \\
c_{2}=\sqrt{3}\left(A_{24}^{2} / 2 \sqrt{7}+A_{20}^{2} / \sqrt{2}+\sqrt{5 / 7} A_{22}^{2}\right), \\
c_{3}=\sqrt{15 / 7}\left(A_{22}^{2}-\sqrt{5} A_{24}^{2} / 4\right), \quad c_{4}=5 \sqrt{21} A_{24}^{2} / 4, \\
c_{5}=\sqrt{15 / 7}\left(\sqrt{5} A_{24}^{2}+3 A_{22}^{2}\right)=3 c_{3}+c_{4}
\end{gathered}
$$

and the four different pseudoscalar parameters

$$
\begin{gathered}
d_{1}=-3\left(A_{23}^{1}+\sqrt{3 / 2} A_{21}^{1}\right), \quad d_{2}=\sqrt{3 / 2} A_{21}^{1}-3 A_{23}^{1} / 2, \\
d_{3}=15 A_{23}^{1} / 2=-3 d_{2}-d_{1}, \\
d_{4}=3 \sqrt{3}\left(\frac{1}{2} \operatorname{Im} A_{23}^{2}+\operatorname{Im} A_{21}^{2}\right), \quad d_{5}=\frac{15}{2} \sqrt{3} \operatorname{Im} A_{23}^{2} .
\end{gathered}
$$

Altogether, as was mentioned at the end of Section 4, there are nine different parameters. From (18) and (20), we obtain

$$
\begin{gathered}
b_{2}^{(0)}=c_{0}\left[3(\mathbf{n p})^{2}-1\right], \quad b_{2}^{(1)}=c_{1}(\mathbf{n p}), \\
b_{2}^{(2)}=c_{2}, \quad b_{2}^{(3)}=c_{3}+c_{4}(\mathbf{n p})^{2}, \\
b_{2}^{(4)}=c_{5}(\mathbf{n p}), \\
a_{2}^{(1)}=-d_{1}(\mathbf{n p}), \quad a_{2}^{(2)}=-d_{3}(\mathbf{n p})^{2}-d_{2}, \\
a_{2}^{(3)}=d_{4}, \quad a_{2}^{(4)}=d_{5}(\mathbf{n p}) .
\end{gathered}
$$

Subtracting from the right-hand side of (19) the righthand side of (17) with $b_{2}^{(j)}$ and $a_{2}^{(j)}$ from (37), we obtain the expression for the quantity $d \sigma_{2} / d \Omega$ for the case of a complete polarization of ionizing radiation defined by the vector e. Here, a more general expression corresponding to the case of a partial polarization is presented, which is obtained by subtraction of the right-hand side of (27) from the right-hand side of (28),

$$
\begin{aligned}
& \frac{d \sigma_{2}(\mathbf{n})}{d \Omega}=c_{0}\left[3(\mathbf{n p})^{2}-1\right]+c_{2}\left[3(\mathbf{k n})^{2}-1\right] / 2 \\
& +\left[c_{3}+c_{4}(\mathbf{n p})^{2}\right]\left[3(\mathbf{k p})^{2}-1\right] / 2 \\
& +c_{5}(\mathbf{n p})[(\mathbf{n p})-3(\mathbf{n k})(\mathbf{k p})] / 2 \\
& +d_{4}(\mathbf{k}[\mathbf{p}, \mathbf{n}])(\mathbf{k n}) / 2+d_{5}(\mathbf{k}[\mathbf{n}, \mathbf{p}])(\mathbf{k p})(\mathbf{n p}) / 2 \\
& +c_{1} \eta_{2}(\mathbf{k}[\mathbf{n}, \mathbf{p}])(\mathbf{n p})+d_{1} \eta_{2}(\mathbf{n p})(\mathbf{k n}) \\
& +\left[d_{2}+d_{3}(\mathbf{n p})^{2}\right] \eta_{2}(\mathbf{k p})+\eta_{1} \delta_{2, p q}+\eta_{3} \delta_{2, x y},
\end{aligned}
$$

where

$$
\begin{gathered}
\delta_{2, i j}=3 c_{2}\left[\left(\mathbf{n e}_{j}\right)^{2}-\left(\mathbf{n e}_{i}\right)^{2}\right] / 2 \\
\left.+3\left[c_{3}+c_{4}(\mathbf{n p})^{2}\right]\left[(\mathbf{p e})_{j}\right)^{2}-\left(\mathbf{p} \mathbf{e}_{i}\right)^{2}\right] / 2 \\
+3 c_{5}(\mathbf{n p})\left[\left(\mathbf{n} \mathbf{e}_{i}\right)\left(\mathbf{p} \mathbf{e}_{i}\right)-\left(\mathbf{n} \mathbf{e}_{j}\right)\left(\mathbf{p e} \mathbf{e}_{j}\right)\right] / 2 \\
+\left\{d_{5}(\mathbf{n p})\left[\mathbf{e}_{i}\left(\mathbf{p} \mathbf{e}_{i}\right)-\mathbf{e}_{j}\left(\mathbf{p} \mathbf{e}_{j}\right)\right]\right. \\
\left.-d_{4}\left[\mathbf{e}_{i}\left(\mathbf{n e}_{i}\right)-\mathbf{e}_{j}\left(\mathbf{n e}_{j}\right)\right]\right\}[\mathbf{p}, \mathbf{n}] / 2 .
\end{gathered}
$$

Using (31) and (32), expression (38) can be transformed to the $(\mathrm{g}, l)$ form. Recall that the pseudoscalars $d_{i}$ differ from zero only for optically active molecules.

In (38), the terms proportional to the Stokes parameter $\eta_{2}$ characterize the CDADP effect arising due to alignment. The achiral CDADP effect upon alignment is determined by the first of these terms, which is proportional to the scalar $c_{1}$. This effect vanishes if photoelectrons escape in the direction perpendicular to the symmetry axis $\mathbf{n}$ of an aligned system, as well as if the vectors $\mathbf{k}, \mathbf{n}$, and $\mathbf{p}$ are coplanar (in particular, if $\mathbf{p}$ and $\mathbf{k}$ are collinear, i.e., upon detection of photoelectrons escaping along or against the direction of propagation of an electromagnetic wave). An additional CDADP effect determined by the chirality of molecules is retained in this experimental geometry (see (38)). This additional effect is also retained if the vectors $\mathbf{k}$ and $\mathbf{p}$ are perpendicular, where the CDADP effect in unpolarized optically inactive molecules vanishes (see (21) and (26)). Consequently, if the vectors $\mathbf{k}, \mathbf{n}$, and $\mathbf{p}$ are coplanar and photoelectrons are detected perpendicularly to the direction of propagation of the electromagnetic wave, the CDADP effect should be observed only due to the alignment of chiral molecules. Note also that, for optically active molecules, this effect depends on the sign of p; i.e., it is somewhat different for photoelectrons moving in opposite directions.

The parameters $c_{1}, d_{4}$, and $d_{5}$ are $T$-odd and, therefore, vanish in the Born approximation. Hence, at large energies of photoelectrons, the CDADP effect is determined precisely by the chirality of aligned molecules (38). Conversely, the dependence of the angular distribution of photoelectrons on the Stokes parameters $\eta_{1}$ and $\eta_{3}$ (i.e., on the degrees of linear polarization of 
radiation) in this energy range is in no way related to the chirality.

Finally, I stress that, as follows from the results obtained in this and preceding sections of this study, some qualitative features of the process of photoionization of oriented and aligned systems are retained in the case of orientation and alignment of an arbitrary order.

In conclusion, I would like to recall that 2005 marks 100 years since Einstein published his famous studies that encompassed three fundamental physical problems. As is known, one of these problems was connected with the photoeffect. The year 2005 was declared the International Year of Physics by the General Assembly of the United Nations Organization. I would like to consider this study as my modest contribution to the celebration of this remarkable anniversary.

APPENDIX

It was shown in [17] that bipolar harmonics (13) of a given rank $r$ can be reduced, i.e., represented as a superposition of the simplest bipolar harmonics of this rank with the minimal possible ranks of internal spherical functions allowed by the selection rules. The coefficients of this superposition are scalar functions of the variable (np) (of the cosine of the angle between the vectors $\mathbf{n}$ and $\mathbf{p}$ ) and are expressed in terms of the Legendre polynomials

$$
P_{K}(x)=\frac{1}{2^{K} K !} \frac{d^{K}}{d x^{K}}\left(x^{2}-1\right)^{K}, \quad x=(\mathbf{n p}),
$$

and their derivatives of the $j$ th order

$$
P_{K}^{(j)}(x)=\frac{d^{j}}{d x^{j}} P_{K}(x),
$$

where $j \leq K$. At $j>K P_{K}^{(j)}=0$.

Here, the reduced expressions for some bipolar harmonics used in this study will be presented for reference purposes. It should be kept in mind that, in (13), the ranks $K$ and $l$ of the tensors and the rank $r$ of the bipolar harmonics satisfy the triangle condition $\Delta(K, l, r)$, from which it follows that $|K-r| \leq l \leq K+r$. This condition imposes some restrictions on the ranks of internal spherical functions in the formulas given below.

The first formula can be obtained using the wellknown summation theorem for spherical functions,

$$
\left\{Y_{K}(\mathbf{n}) \otimes Y_{K}(\mathbf{p})\right\}_{0}=(-1)^{K} \frac{\sqrt{2 K+1}}{4 \pi} P_{K}(\mathbf{n p}) .
$$

The next four formulas were presented in the appendix of [17]:

$$
\begin{gathered}
\left\{Y_{K}(\mathbf{n}) \otimes Y_{K}(\mathbf{p})\right\}_{1} \\
=i \frac{(-1)^{K+1}}{4 \pi}\left[\frac{3(2 K+1)}{K(K+1)}\right]^{1 / 2} P_{K}^{(1)}(\mathbf{n p})[\mathbf{n}, \mathbf{p}],
\end{gathered}
$$

where $K=1,2,3, \ldots$;

$$
\begin{aligned}
& \left\{Y_{K}(\mathbf{n}) \otimes Y_{K \pm 1}(\mathbf{p})\right\}_{1} \\
= & \frac{(-1)^{K+1}}{4 \pi} \sqrt{3}\left(K+\frac{1}{2} \pm \frac{1}{2}\right)^{-1 / 2} \\
\times & {\left[P_{K}^{(1)}(\mathbf{n p}) \mathbf{n}-P_{K \pm 1}^{(1)}(\mathbf{n p}) \mathbf{p}\right], }
\end{aligned}
$$

where, at $K=0$, only one bipolar harmonic with the rank of the second spherical function equal to $K+1=1$ remains;

$$
\begin{gathered}
\left\{Y_{K}(\mathbf{n}) \otimes Y_{K \pm 1}(\mathbf{p})\right\}_{2} \\
=i \frac{(-1)^{K}}{4 \pi} \sqrt{10}\left[K(K \pm 1)\left(K+\frac{3}{2} \pm \frac{1}{2}\right)\right]^{-1 / 2} \\
\times\left[P_{K}^{(2)}(\mathbf{n p})\{\mathbf{n} \otimes[\mathbf{n}, \mathbf{p}]\}_{2}\right. \\
\left.+P_{K \pm 1}^{(2)}(\mathbf{n p})\{\mathbf{p} \otimes[\mathbf{p}, \mathbf{n}]\}_{2}\right],
\end{gathered}
$$

where $K=1,2,3, \ldots$ and, at $K=1$, only one bipolar harmonic with the rank of the second spherical function equal to $K+1=2$ remains; and

$$
\begin{gathered}
\left\{Y_{K}(\mathbf{n}) \otimes Y_{K \pm 2}(\mathbf{p})\right\}_{2} \\
=\frac{(-1)^{K}}{4 \pi} \sqrt{5}[(K+1 \pm 1)(2 K+1 \pm 2)(K \pm 1)]^{-1 / 2} \\
\times\left[P_{K}^{(2)}(\mathbf{n p})\{\mathbf{n} \otimes \mathbf{n}\}_{2}-2 P_{K \pm 1}^{(2)}(\mathbf{n p})\{\mathbf{n} \otimes \mathbf{p}\}_{2}\right. \\
\left.+P_{K \pm 2}^{(2)}(\mathbf{n p})\{\mathbf{p} \otimes \mathbf{p}\}_{2}\right],
\end{gathered}
$$

where, at $K=0$ and 1 , only the bipolar harmonic with the rank of the second spherical function equal to $K+2$ remains. Finally, the latter bipolar harmonic was reduced directly according to the method of [17] since, in the corresponding expression, which was presented in the appendix to the cited paper, the procedure of reduction was not accomplished. The result is as follows:

$$
\begin{gathered}
\left\{Y_{K}(\mathbf{n}) \otimes Y_{K}(\mathbf{p})\right\}_{2} \\
=\frac{(-1)^{K}}{4 \pi}\left[\frac{30(2 K+1)}{(2 K-1) K(K+1)(2 K+3)}\right]^{1 / 2} \\
\times\left[P_{K}^{(2)}(\mathbf{n p})\left(\{\mathbf{n} \otimes \mathbf{n}\}_{2}+\{\mathbf{p} \otimes \mathbf{p}\}_{2}\right)\right. \\
\left.+\left((2 K+3) P_{K}^{(1)}(\mathbf{n p})-P_{K+1}^{(2)}(\mathbf{n p})\right)\{\mathbf{n} \otimes \mathbf{p}\}_{2}\right],
\end{gathered}
$$

where $K=1,2,3, \ldots$.

\section{REFERENCES}

1. C. N. Yang, Phys. Rev. 74 (7), 764 (1948).

2. M. Ya. Amus'ya, The Photoelectric Effect in Atoms (Nauka, Moscow, 1987) [in Russian].

3. N. A. Cherepkov, Adv. At. Mol. Phys. 19, 395 (1983).

4. C. M. Lee, Phys. Rev. A 10 (5), 1598 (1974).

5. U. Heinzmann, J. Phys. B 13 (22), 4367 (1980). 
6. B. Schmidtke, M. Dresher, N. A. Cherepkov, and U. Heinzmann, J. Phys. B 33 (13), 2451 (2000).

7. N. A. Cherepkov and S. K. Semenov, J. Phys. B 37 (6), 1267 (2004).

8. H. Klar and H. Kleinpoppen, J. Phys. B 15 (6), 933 (1982).

9. G. V. Skrotskiı̆ and T. G. Izyumova, Usp. Fiz. Nauk 73 (1961) [Sov. Phys. Usp. 4, 177 (1961)].

10. W. Happer, Rev. Mod. Phys. 44 (2), 169 (1972).

11. K. Blum, Density Matrix Theory and Applications (Plenum, New York, 1996).

12. D. A. Varshalovich, A. N. Moskalev, and V. K. Khersonskii (Khersonskiǔ), Quantum Theory of Angular Momentum (Nauka, Leningrad, 1975; World Scientific, Singapore, 1988).

13. N. A. Cherepkov and V. V. Kuznetsov, J. Phys. B 22 (14), L405 (1989).

14. V. D. Ovsyannikov, Opt. Spektrosk. 75 (1), 24 (1993) [Opt. Spectrosc. 75 (1), 14 (1993)].

15. S. Baier, A. N. Grum-Grzimailo, and N. M. Kabachnik, J. Phys. B 27 (15), 3363 (1994).

16. N. A. Cherepkov, V. V. Kuznetsov, and V. A. Verbitskii, J. Phys. B 28 (7), 1221 (1995).

17. N. L. Manakov, S. I. Marmo, and A. V. Meremianin, J. Phys. 29 (13), 2711 (1996).
18. M. Ya. Agre, Zh. Éksp. Teor. Fiz. 122, 233 (2002) [JETP 95, 199 (2002)].

19. M. Ya. Agre, Opt. Spektrosk. 94 (2), 193 (2003) [Opt. Spectrosc. 94 (2), 163 (2003)].

20. B. Ritchie, Phys. Rev. A 14 (1), 359 (1976).

21. N. L. Manakov, Zh. Éksp. Teor. Fiz. 106, 1286 (1994) [JETP 79, 696 (1994)].

22. M. Ya. Agre and N. L. Manakov, J. Phys. B 29 (1), L7 (1996).

23. M. Ya. Agre, Zh. Éksp. Teor. Fiz. 110, 2018 (1996) [JETP 83, 1112 (1996)].

24. L. D. Landau and E. M. Lifshitz (Lifshits), The Classical Theory of Fields, 4th English ed. (Nauka, Moscow, 1973; Pergamon Press, Oxford, 1975).

25. M. Ya. Agre, Zh. Éksp. Teor. Fiz. 120, 562 (2001) [JETP 93, 491 (2001)].

26. V. B. Berestetskii, E. M. Lifshitz (Lifshits), and L. P. Pitaevskii, Quantum Electrodynamics, 2nd ed. (Nauka, Moscow, 1980; Pergamon Press, Oxford, 1982).

27. S. J. Schaphorst, B. Krässig, O. Schwarzkopf, et al., J. Electron Spectrosc. Relat. Phenom. 76, 229 (1995).

Translated by V. Rogovo 